\title{
Effects of Dexamethasone Treatment on Bone and Collagen Turnover in Preterm Infants with Chronic Lung Disease
}

\author{
PATRICIA M. CROFTON, ANOUPAM SHRIVASTAVA, JEAN C. WADE, RHONA STEPHEN, \\ CHRISTOPHER J.H. KELNAR, NEIL MCINTOSH, AND ANDREW J. LYON
}

Department of Paediatric Biochemistry, Royal Hospital for Sick Children, Edinburgh, United Kingdom [P.M.C.]; Department of Child Life and Health, University of Edinburgh, Edinburgh, United Kingdom [P.M.C., A.S., J.C.W., R.S., C.J.H.K., N.M., A.J.L.]; and Simpson Memorial Maternity Pavilion, Royal Infirmary, Edinburgh, United Kingdom [N.M., A.J.L.]

\begin{abstract}
ABST
Dexamethasone is used commonly in the treatment of chronic
lung disease of prematurity, but there are concerns about possible
deleterious effects on growth and bone. Our aim in this study was
to examine the effects of dexamethasone treatment on bone and
collagen turnover in preterm infants. Bone-specific alkaline
phosphatase, the C-terminal propeptide of type I collagen (PICP,
reflecting whole-body type I collagen synthesis), and the N-
terminal propeptide of type III procollagen (P3NP, reflecting soft
tissue collagen turnover), together with the C-terminal telopep-
tide of type I collagen (ICTP), urinary pyridinoline (Pyd), and
deoxypyridinoline (all markers of collagen breakdown) were
measured at weekly intervals over the first 12 wk of life in 14
preterm infants with chronic lung disease treated with dexameth-
asone. Results were expressed as SD scores relative to preterm
control infants not treated with dexamethasone. PICP, P3NP,
ICTP, and Pyd all showed marked decreases ( -2.1 to $-3.7 \mathrm{SD}$
scores) during the first week of treatment ( $p<0.001)$, returning
to pretreatment levels after stopping dexamethasone. In the group
as a whole, these collagen markers were negatively correlated
with dexamethasone dose $(p<0.0001)$; negative correlations
\end{abstract}
Steroids are used commonly in the management of preterm infants with developing chronic lung disease (CLD). Dexamethasone has been shown to improve pulmonary compliance and help to wean infants from the ventilator, but there are concerns about side effects (1-3). Glucocorticoids impair growth and bone formation in children (4-8). We and others have demonstrated that infants treated with dexamethasone have short-term reductions in linear growth, weight velocity, radial length velocity, and bone mineralization compared with those not so treated $(9-11)$. We have also demonstrated that these infants have reduced calcium absorption and retention,

Received June 8, 1999; accepted February 2, 2000.

Correspondence: Dr. P.M. Crofton, Department of Paediatric Biochemistry, Royal Hospital for Sick Children, Sciennes Road, Edinburgh EH9 1LF, United Kingdom. were also seen in most individual babies, although the slopes of individual regression lines varied by a factor of 2 . Weight gain at 12 wk was correlated with PICP, expressed as the mean SD score over 12 wk for each baby, $(r=0.69, p<0.01)$ but not with other markers or cumulative dose of dexamethasone. We conclude that dexamethasone markedly suppressed collagen turnover in preterm infants in a dose-dependent fashion, although some babies were more affected than others. The degree of suppression of type I collagen synthesis was a strong independent predictor of overall weight gain over the first 12 wk of life. (Pediatr Res 48: 155-162, 2000)

Abbreviations
CLD, chronic lung disease
ALP, alkaline phosphatase
PICP, C-terminal propeptide of type I collagen
P3NP, N-terminal propeptide of type III collagen
ICTP, the cross-linked telopeptide of type I collagen
Pyd, pyridinoline
Dpd, deoxypyridinoline

and reduced phosphate retention, compared with infants who did not receive steroids (11).

Biochemical markers of bone and soft tissue turnover may give insight into the dynamic effects of therapeutic interventions on bone and growth (12). For the present study, we chose a panel of markers that would reflect various aspects of bone and soft tissue turnover and that have already been validated as markers of growth in older children and as markers of bone formation and resorption by histomorphometry and calcium kinetic studies in adults (12). Bone alkaline phosphatase (ALP) is a marker of the differentiated osteoblast and also of hypertrophic chondrocytes in the growth plate. The C-terminal propeptide of type I collagen (PICP) quantitatively reflects type I collagen synthesis; it is produced principally by the osteoblast in its proliferative phase but may also, to a lesser extent, reflect 
type I collagen synthesis elsewhere in the body. The Nterminal propeptide of type III collagen (P3NP) is purely a marker of type III collagen synthesis in soft tissue. The crosslinked telopeptide of type I collagen (ICTP) reflects the degradation of type I collagen, largely, but not exclusively, of bone origin. Pyridinoline (Pyd), measured in urine, reflects whole body collagen degradation (excluding that in skin) whereas deoxypyridinoline (Dpd) is thought to be more specific for bone collagen. We have already demonstrated that in preterm infants not treated with glucocorticoids, PICP and P3NP increase after birth, whereas ICTP decreases, reflecting postnatal growth (13). P3NP was correlated with rate of weight and length gain in these infants, whereas PICP was highly correlated with bone mineral content.

We report here a longitudinal prospective study on a separate group of preterm infants treated with high-dose dexamethasone. Our aim was to explore its effects on bone and soft tissue turnover in these infants, to determine whether these effects were dose-related, and to examine their relationship with growth over the first 12 wk of life.

\section{PATIENTS AND METHODS}

\section{Subjects}

All infants admitted sequentially to the neonatal intensive care unit over a 15-mo period from August 1993 to November 1994 with a birthweight less than $1500 \mathrm{~g}$, gestational age at birth less than $34 \mathrm{wk}$, and who were treated with dexamethasone for developing CLD were eligible to participate in the study. CLD was defined retrospectively as persisting oxygen dependence at $28 \mathrm{~d}$ postnatal age in the presence of an abnormal chest radiograph. The decision to treat with dexamethasone was made on clinical grounds by the team caring for the infant. The policy on the unit at that time was to start dexamethasone during the third postnatal week if an infant could not be weaned from the ventilator and had signs of developing CLD on chest radiograph. Dexamethasone was commenced at $500 \mu \mathrm{g} / \mathrm{kg}$ body weight/d for $3 \mathrm{~d}$, followed by gradually decreasing doses for a total treatment period of $3 \mathrm{wk}$. There was, however, some variation in the timing and duration of dexamethasone treatment, for clinical reasons. Subsequent courses of dexamethasone were used as clinically indicated. In practice, all infants in this study who were treated with dexamethasone had CLD, as assessed at $28 \mathrm{~d}$ postnatal age.

All infants were fed on mother's breast milk with preterm formula (Prematil, Milupa, Trowbridge, U.K.) added if the breast milk supply was inadequate. Oral vitamin D supplements of $1000 \mathrm{U} / \mathrm{d}$ were started once the infant was tolerating full enteral feeds. Phosphate supplements $(1 \mathrm{mmol} / \mathrm{d}$ as sodium hydrogen phosphate) were given if the plasma phosphate concentration fell below $1.5 \mathrm{mM}$.

\section{Measurements}

Body weight was measured at a minimum of weekly intervals in all infants. Length measurements were also made in 9 of the 14 infants who could tolerate the procedure. We collected blood and urine samples weekly for $12 \mathrm{wk}$ in all infants, during mid-morning to minimize the effect of any circadian variation on the markers of bone turnover. In addition, we collected an extra blood and urine sample $3 \mathrm{~d}$ after commencing dexamethasone. Samples were stored at $-70^{\circ} \mathrm{C}$ until analysis. The study was approved by the local ethics committee and informed parental consent was obtained in all cases.

\section{Analytical Methods}

Collagen assays. PICP, P3NP, and ICTP were measured in plasma by RIA (Orion Diagnostica, Espoo, Finland), using methods previously described (14-16). Before analysis, we diluted samples appropriately in $154 \mathrm{mM}$ sodium chloride to achieve concentrations within the calibration curve; typical dilutions were 1 in 30 for PICP and 1 in 20 for P3NP and ICTP. All samples were analyzed in duplicate. As far as possible, samples from each infant were analyzed in a single run to minimize analytical variation. Between-run coefficients of variation for manufacturer's controls were $7.8 \%$ and $5.2 \%$ at 94 $\mu \mathrm{g} / \mathrm{L}$ and $320 \mu \mathrm{g} / \mathrm{L}$ for PICP, $5.6 \%$ at $4.6 \mu \mathrm{g} / \mathrm{L}$ for P3NP, and $6.3 \%$ and $9.2 \%$ at $8.7 \mu \mathrm{g} / \mathrm{L}$ and $33.8 \mu \mathrm{g} / \mathrm{L}$ for ICTP. For pooled plasma from preterm infants, diluted as above, they were $4.4 \%$ at $3990 \mu \mathrm{g} / \mathrm{L}$ for PICP, $6.4 \%$ at $208 \mu \mathrm{g} / \mathrm{L}$ for P3NP, and $11.7 \%$ at $118 \mu \mathrm{g} / \mathrm{L}$ for ICTP.

Bone ALP. Bone ALP was measured in plasma by an ELISA (Alkphase-B ${ }^{\mathbb{T N}}$ from Metra Biosystems Inc., Mountain View, CA, U.S.A.). The assay uses a monoclonal anti-bone ALP antibody coated on a microtitre plate to capture bone ALP in the samples. This assay gives approximately $10 \%$ crossreactivity with the liver isoform, but this is not present in plasma from preterm infants (17). Using plasma samples from preterm infants containing high activities of fetal intestinal ALP, and also amniotic fluid and extracts of meconium from preterm infants (both of which contain only fetal intestinal ALP), we confirmed that the assay gave no cross-reaction with fetal intestinal ALP, which may comprise up to half the total plasma ALP activity in preterm infants (18). Before analysis, we diluted samples 1 in 3 in $154 \mathrm{mM}$ sodium chloride to achieve concentrations within the calibration curve. Betweenrun coefficients of variation for manufacturer's controls were $6.7 \%$ and $5.4 \%$ at $15.1 \mathrm{U} / \mathrm{L}$ and $70.5 \mathrm{U} / \mathrm{L}$, respectively. For pooled plasma from preterm infants, diluted as above, it was $6.6 \%$ at $126 \mathrm{U} / \mathrm{L}$.

Pyridinium cross-links. Pyridinium cross-links were measured in urine by HPLC using a modification of the method of Pratt et al. (19). In urine, approximately $40 \%$ of pyridinium cross-links are in the unconjugated (free) form whereas the remainder are either glycosylated or present as low molecular weight peptides. The urine samples were hydrolyzed to convert the glycosylated and peptide forms to the free form and, after solid phase extraction using CC31 microgranular cellulose, total Pyd and Dpd were measured by isocratic reversed-phase HPLC, using a C-8 $(4.6 \times 250 \mathrm{~mm})$ column, heptafluorobutyric acid as the ion-pairing agent, and acetonitrile as the organic modifier in the mobile phase. Pyridinium cross-links extracted from demineralized sheep bone were used as external standards (20) and were a generous gift from Dr. Simon Robins (Rowett Research Institute, Aberdeen, Scotland). The results 

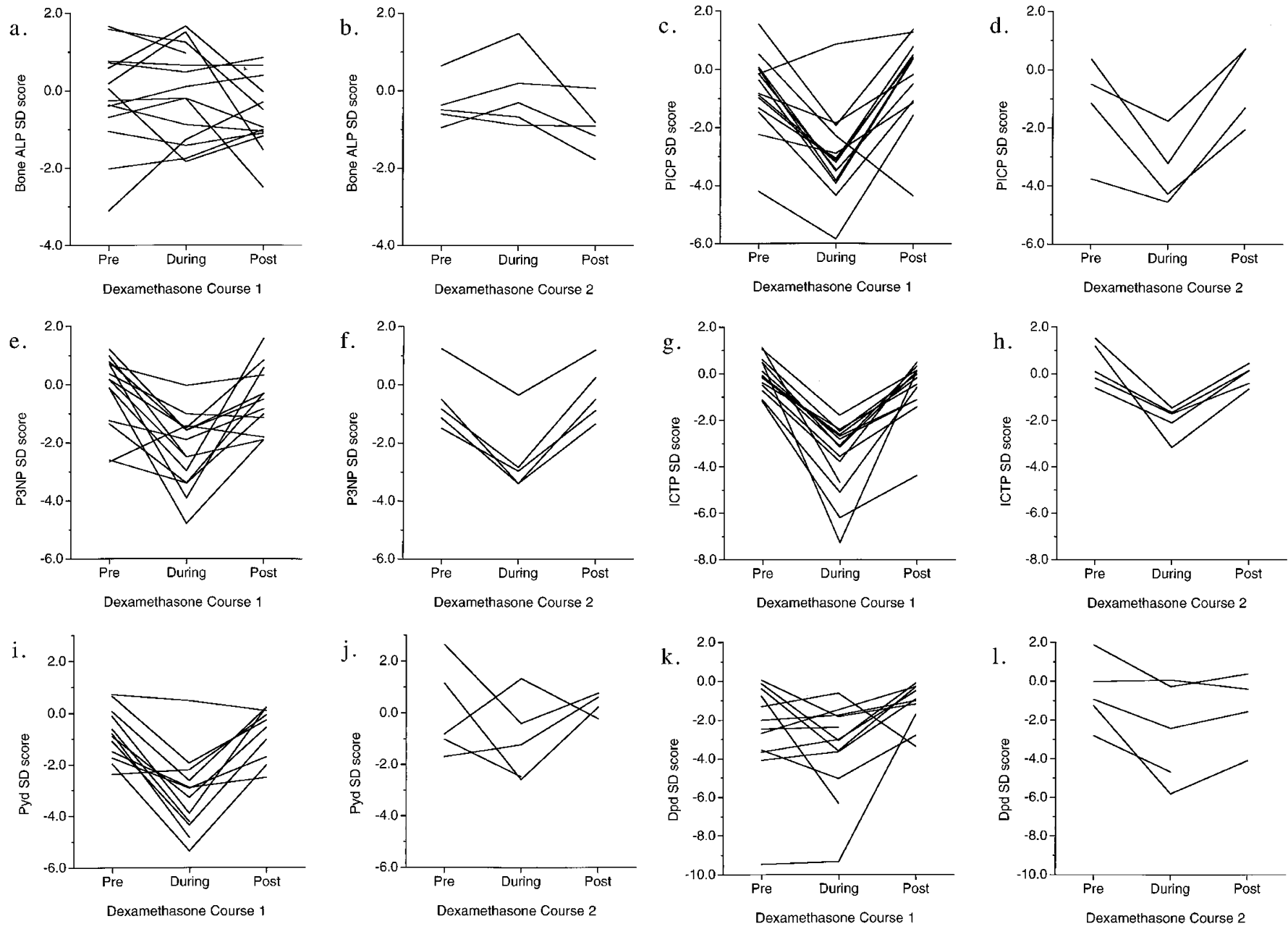

Figure 1. Marker SD scores for individual babies during the first week of dexamethasone treatment, compared with the week before starting treatment and the first week after the end of the treatment course. (a, b) Bone ALP, (c, d) PICP, (e, f) P3NP, (g, h) ICTP, (i, j) Pyd, (k, 1) Dpd. (a, c, e, g, i, k) First treatment course, $(b, d, f, h, j, l)$ second treatment course.

were expressed in relation to creatinine measured on the same urine sample. Samples were assayed in duplicate with mean interassay coefficients of variation of $5.9 \%$ at $315 \mathrm{nmol} / \mathrm{mmol}$ creatinine for Pyd and $9.1 \%$ at $65.8 \mathrm{nmol} / \mathrm{mmol}$ creatinine for Dpd. The interassay coefficient of variation for the ratio of Pyd to Dpd was $7.9 \%$ at a ratio of 4.8 .

\section{Data Analysis}

All biochemical marker concentrations were positively skewed and were therefore $\log$ transformed to fit a gaussian distribution and equalize variances. Because there are rapid changes after birth in many of these markers (13), we converted them to SD scores in relation to control infants matched for postnatal age. The controls were preterm infants of similar gestational age and birthweight who were born in the same unit over the same time period but did not receive steroids. These controls have been fully described elsewhere (13). Briefly, they had a median birthweight of $1115 \mathrm{~g}$ (range 784-1505 g) and a median gestational age of $28 \mathrm{wk}$ (range 24-33 wk). Half of the control babies developed CLD (although they were not given steroids) but this had no apparent effect on their marker concentrations. Data from all the controls were therefore used to derive SD scores for our study infants. Conversion to SD scores in this manner allowed us to compare infants who started dexamethasone at different postnatal ages and had different durations of treatment. It also facilitated comparison between the different markers in terms of the magnitude of their response to dexamethasone treatment.

We used parametric statistics throughout. All statistical tests were two-tailed and $p<0.05$ was regarded as significant. Unless otherwise stated, data (after conversion to SD scores, as described above) were expressed as mean and $95 \%$ confidence intervals for the mean. Comparisons of the study group and controls were by the one-sample $t$ test (the mean SD score of the control population being zero by definition). Changes through time were tested by ANOVA, using Fisher's protected least significant difference as a post hoc test. Among those infants who had two courses of dexamethasone treatment, marker responses to each course were compared using paired $t$-tests. Simple linear regression was used to assess relations between variables.

Overall weight gain at 84 postnatal d (12 wk) was calculated by interpolation between the weight measurements immediately before and after d 84, followed by subtraction of the birthweight. 
Rate of length gain was assessed in each infant by linear regression of all length measurements over the first $12 \mathrm{wk}$ of life. To explore relations between the markers and overall weight gain or linear growth, we calculated, for each marker in each baby, its overall mean SD score over the first $12 \mathrm{wk}$ of life, equivalent to the area under the curve. To evaluate which variable(s) contributed most to the anthropometric outcomes of (1) weight gain at 12 wk and (2) rate of linear growth, we constructed multiple regression models using forward stepwise regression, with reiterative evaluation to remove noncontributory variables (partial $F$-to-enter and $F$-to-remove 3.953, equivalent to a significance level of $p<$ $0.05)$. The variables introduced to each model were birthweight, cumulative dexamethasone dose, and the infant's overall mean SD score for each marker. The proportion of the variance in (1) weight gain or (2) rate of length gain predicted by each model was estimated using the adjusted $r^{2}$ to compensate for the number of variables entered.

\section{RESULTS}

The median birthweight of the 14 babies enrolled in the study was $814 \mathrm{~g}$ (range 578-1150 g) and their median gestational age at birth was 27 wk (range 23-29 wk). The first course of dexamethasone was started at a median postnatal age of $20 \mathrm{~d}$ (range 13-50 d) and lasted for a median duration of $25 \mathrm{~d}$ (range 3-78 d). Five babies had a second course of dexamethasone, separated from the first course by a minimum of $10 \mathrm{~d}$; this was started at a median postnatal age of $50 \mathrm{~d}$ (range 34-73 d) and lasted for a median duration of $12 \mathrm{~d}$ (range 2-24 d).

\section{Before Dexamethasone Treatment}

During the first week of life, mean SD scores for the group (95\% confidence intervals, $p$ value compared with controls) were: bone ALP $-0.88(-1.75,0.00, p<0.05)$, PICP 0.49 $(0.10,0.89, p<0.05)$, P3NP $-0.39(-0.98,0.20, \mathrm{NS})$, ICTP $-0.11(-0.63,0.40$, NS), Pyd $-1.17(-1.61,-0.72, p<$ $0.001)$, and Dpd $-2.00(-4.07,0.06, \mathrm{NS})$, respectively. From the first week of life to the week immediately preceding dexamethasone treatment, there was an upward trend in bone ALP and a downward trend in PICP (both $p<0.05$ ), but no statistically significant change in any other marker.

\section{Dexamethasone Treatment}

Figure 1, a- 1 , and Table 1 show the marker SD scores during the first week of dexamethasone treatment, compared with the week before starting treatment and the first week after the end

Table 1. Group mean SD scores (95\% confidence intervals) for each marker during the week before starting dexamethasone, the first week of treatment, and the week after stopping treatment

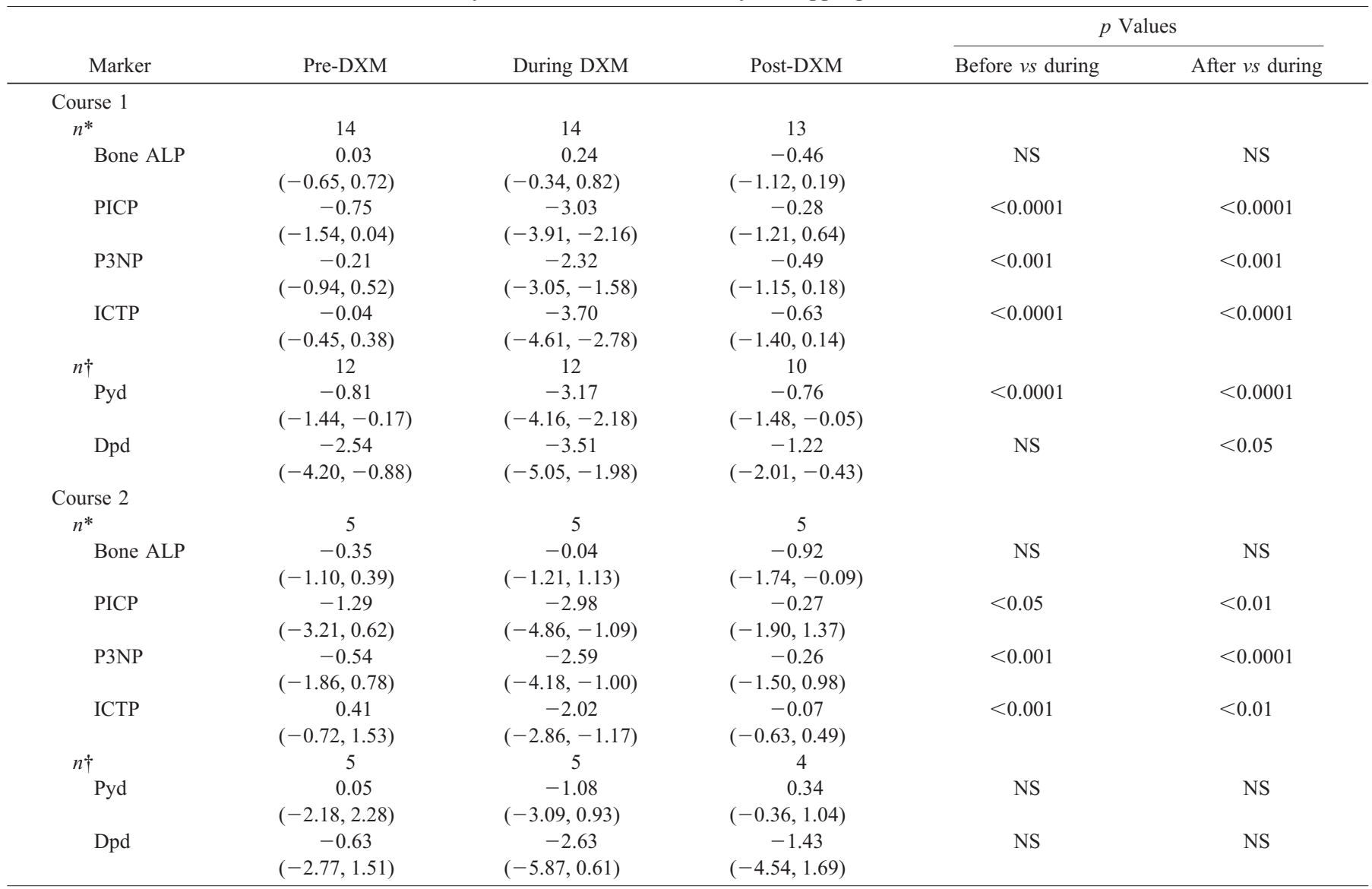

Paired comparisons were by Fisher's protected least significant differences following ANOVA. There were no significant differences between pre- and post-DXM for any of the markers. DXM, dexamethasone; NS, not significant.

* Number of babies from whom plasma samples were collected.

$\dagger$ Number of babies from whom urine samples were collected. 
of the treatment course. Among those babies who had two courses of treatment, there was no difference between the courses in terms of marker response, except that Pyd excretion decreased by a greater amount during the first course than the second $(p=0.004)$. For the first course of treatment, ANOVA indicated marked changes through time for PICP, P3NP, ICTP, Pyd (all $p<0.0001$ ), and, to a lesser extent, for Dpd ( $p=$ $0.033)$, but not for bone ALP $(p=0.13)$. For the five babies who had a second course of treatment, there were still significant changes through time for PICP, P3NP, and ICTP $(p<$ 0.01 ), but not for the other markers. The mean changes in SD scores $(95 \%$ confidence intervals) within $1 \mathrm{wk}$ of starting the first course of dexamethasone were bone ALP $0.21(-0.23$, $0.65)$, PICP $-2.28(-3.08,-1.49)$, P3NP $-2.10(-3.04$, $-1.17)$, ICTP $-3.66(-4.56,-2.76)$, Pyd -2.37 (-3.26, $-1.48)$ and Dpd $-0.97(-2.28,0.34)$. The proportion of dexamethasone courses in which the markers decreased during the first week of treatment were bone ALP 8/19, PICP 18/19, P3NP 18/19, ICTP 19/19, Pyd 17/19, and Dpd 12/19.

\section{Relationship Between Markers and Dexamethasone Dose}

During dexamethasone treatment, for all infants combined (excluding the infant who received dexamethasone for only $3 \mathrm{~d}$ ), the collagen markers were strongly and negatively correlated with the mean dexamethasone dose over the previous $3 \mathrm{~d} ; r$ values were -0.57 for PICP, -0.58 for P3NP,-0.69 for ICTP, -0.48 for Pyd (all $p<0.0001$ ), and -0.26 for $\operatorname{Dpd}(p=$ 0.005 ) (Fig. 2, a-e). Bone ALP was not significantly correlated with dexamethasone dose $(r=0.15, p=0.90)$. Table 2 shows the correlations for each individual baby. Median within-baby $r$ values were bone ALP 0.36 , PICP -0.79 , P3NP -0.70 , ICTP -0.74 , Pyd -0.58 , and Dpd -0.23 . It is clear that the plasma markers of collagen turnover (PICP, P3NP, and ICTP) were generally strongly and negatively correlated with mean dexamethasone dose but that there were individual babies in whom the relationship was less strong. Urinary markers of collagen degradation (Pyd and Dpd) had more variable, but largely negative relationships with dexamethasone dose. Bone ALP had very variable and generally nonsignificant positive relationships with dexamethasone dose. Among those babies who had strong negative correlations between PICP, P3NP, ICTP, Pyd, and dexamethasone dose, the slopes of the regression lines varied by a factor of two for the same marker, suggesting that babies differed in terms of the effect of dexamethasone on their collagen turnover.

\section{Correlations Among Markers}

The correlations among markers (expressed as mean SD score for each baby over the first $12 \mathrm{wk}$ of life) are given in Table 3. Most of the markers of collagen turnover were correlated with one another to varying extents but bone ALP was correlated only weakly to Pyd and not to the other markers.

\section{Relationship of Markers with Anthropometric Variables}

Thirteen babies survived to week 12. For these infants, median weight gain at $\mathrm{d} 84$ was $1621 \mathrm{~g}$ (range 1068-2233 g).
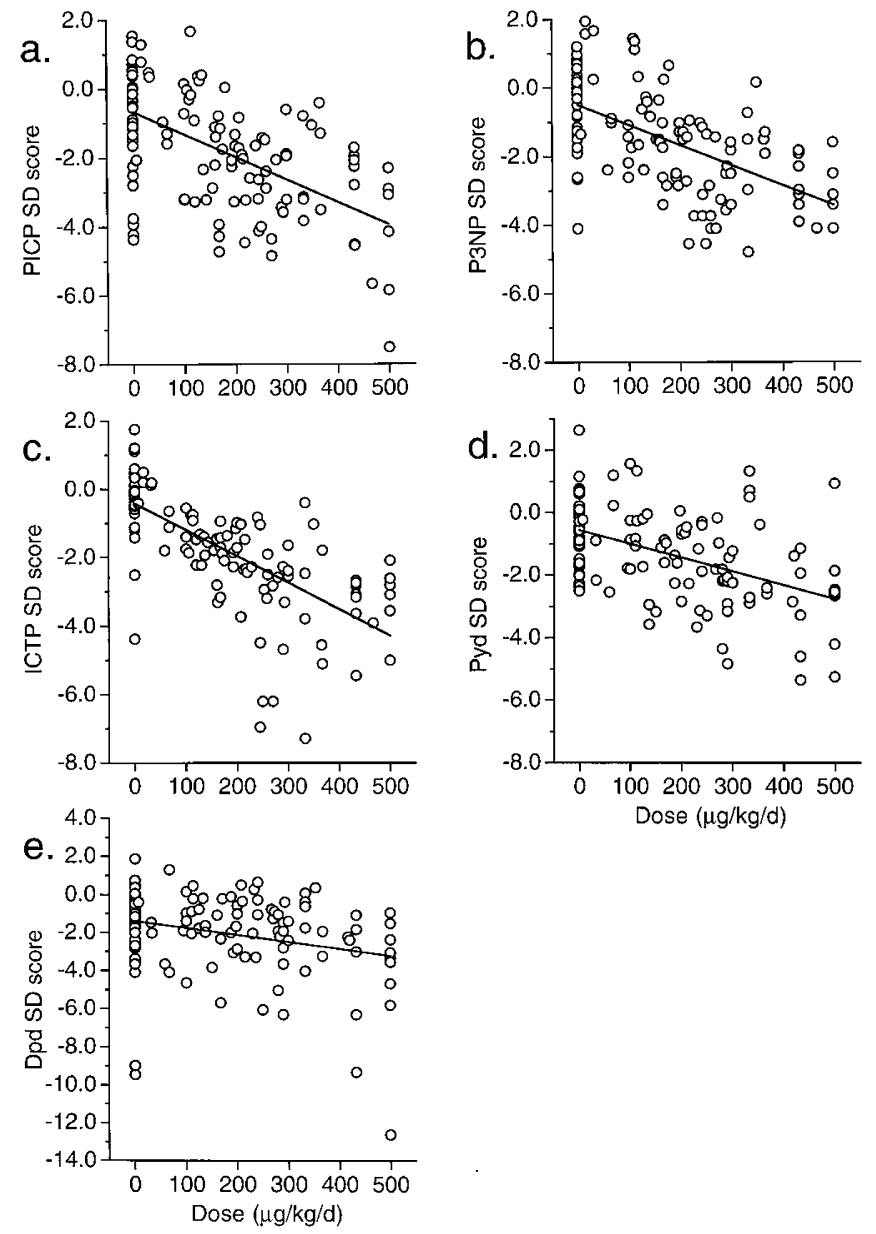

Figure 2. Collagen marker SD scores versus the mean dexamethasone dose over the previous 3 d. (a) PICP, (b) P3NP, (c) ICTP, (d) Pyd, (e) Dpd.

This was weakly related to birthweight $(r=0.55, p=0.05)$ but not to the cumulative dose of dexamethasone $(r=0.01$, NS). However, PICP (expressed as mean SD score over the first $12 \mathrm{wk}$ of life) was positively correlated to weight gain at d $84(r=0.69, p<0.01)$ (Fig. 3). There was no significant correlation between weight gain and any other marker. Multiple regression with stepwise selection of variables confirmed that only PICP contributed independently to the prediction of weight gain, explaining $47 \%$ of its variance. Neither birthweight nor cumulative dexamethasone dose were contributory.

For the nine infants in whom we measured length, the median rate of length gain was $1.26 \mathrm{~cm} / \mathrm{wk}$ (range $0.77-1.54$ $\mathrm{cm} / \mathrm{wk}$ ). Rate of length gain was correlated positively with PICP (expressed as mean SD score over the first $12 \mathrm{wk}$ of life) $(r=0.59, p=0.09)$ and negatively with that for Dpd $(r=$ $-0.61, p=0.08$ ), although these correlations failed to reach statistical significance owing to the small numbers. Birthweight and cumulative dexamethasone dose showed no relationship with linear growth. Multiple regression with stepwise selection of variables identified Dpd as the only independent variable that contributed significantly to the prediction of linear growth, although it explained only $28 \%$ of its variance. Again, neither birthweight nor cumulative dexamethasone dose were contributory. 
Table 2. Correlation coefficients in individual babies between markers and mean dexamethasone dose received by that baby over the previous $3 d$

\begin{tabular}{|c|c|c|c|c|c|c|c|c|}
\hline Infant & $n^{*}$ & Bone ALP & PICP & P3NP & ICTP & $n \dagger$ & Pyd & Dpd \\
\hline 2 & 9 & 0.59 & $-0.68 \$$ & $-0.76 \$$ & $-0.74 \downarrow$ & 7 & 0.05 & -0.07 \\
\hline 3 & 14 & -0.09 & $-0.82 \S$ & $-0.87 \S$ & $-0.92 \S$ & 13 & -0.78 & $-0.60 \%$ \\
\hline 4 & 8 & 0.49 & $-0.49^{\circ}$ & $-0.51^{\circ}$ & $-0.93 \S$ & 8 & 0.30 & 0.05 \\
\hline 6 & 9 & $0.69 \ddagger$ & -0.819 & -0.16 & $-0.62 \ddagger$ & 8 & -0.58 & -0.27 \\
\hline 7 & 9 & $0.65^{\circ}$ & $-0.91 \S$ & $-0.81 \Phi$ & $-0.93 \S$ & 9 & -0.45 & -0.29 \\
\hline 8 & 12 & 0.36 & -0.799 & -0.73 & $-0.59 \ddagger$ & 13 & -0.64 & 0.01 \\
\hline 11 & 5 & -0.66 & -0.80 & -0.86 & -0.55 & 6 & -0.85 & -0.23 \\
\hline 12 & 12 & 0.48 & -0.19 & -0.49 & $-0.85 \S$ & 12 & $-0.63+$ & -0.49 \\
\hline 13 & 6 & 0.34 & $-0.86 \$$ & -0.70 & -0.979 & 5 & 0.11 & 0.01 \\
\hline 14 & 5 & 0.20 & $-0.91 \ddagger$ & -0.71 & $-0.93 \ddagger$ & 4 & -0.93 & -0.62 \\
\hline
\end{tabular}

* Number of blood samples collected during dexamethasone treatment in that baby.

$\dagger$ Number of urine samples collected during dexamethasone treatment in that baby.

$\ddagger p<0.05$.

$\S p<0.001$

\ $p<0.01$.

|| Baby died just after the last blood sample.

Table 3. Correlations among markers (expressed as mean SD score for each baby over the first 12 wk of life)

\begin{tabular}{lllccc}
\hline & PICP & P3NP & ICTP & Pyd & Dpd \\
\hline Bone ALP & -0.02 & 0.08 & -0.06 & $0.19^{*}$ & 0.17 \\
PICP & & $0.73 \dagger$ & $0.60 \dagger$ & $0.32 \dagger$ & $0.41 \dagger$ \\
P3NP & & & $0.64 \dagger$ & $0.48 \dagger$ & $0.24 \dagger$ \\
ICTP & & & & 0.17 \\
Pyd & & & & $0.57 \dagger$ \\
\hline
\end{tabular}

$* p<0.05$.

$\dagger p<0.001$.

$\ddagger p<0.01$.

\section{DISCUSSION}

The optimum dose and duration of dexamethasone treatment required to achieve a clinical effect with the minimum of side effects has not been determined. We and others have observed short-term reductions in weight gain, linear growth, and lower leg growth in infants treated with high-dose dexamethasone $(9-11)$. Studies of outcome at $2-3$ y of age have reached different conclusions regarding longer-term effects on growth $(2,3)$. There is little information available concerning the mechanism of the adverse effects of dexamethasone, whether these are dose-related and whether individual babies differ in their susceptibility to these side-effects. The present study was designed to explore these aspects as a necessary preliminary to a formal double-blind randomized trial of different dexamethasone dosages and regimes on longer-term outcome. Our study was therefore essentially observational, using a conventional high-dose dexamethasone treatment regime with tapering doses determined on clinical grounds. The infants acted largely as their own controls although data were expressed as SD scores in relation to nonsteroid-treated control preterm infants, partly to correct for the normal postnatal changes that occur in markers of bone and collagen turnover (13) and partly to facilitate comparisons between markers.

We found that before dexamethasone treatment began, both Pyd and Dpd were lower than in controls but showed no evidence of any trend through time. By contrast, bone ALP was slightly lower and PICP was slightly higher than in controls during the first week of life; bone ALP then increased to normal whereas PICP decreased before steroids were started. Some of these changes may have reflected the clinical state of the babies before dexamethasone treatment began, although neither P3NP nor ICTP differed from the controls before steroid treatment, nor did they show any progressive trend.

During the first week after starting dexamethasone, there was a rapid and profound decrease in most markers of collagen turnover, in contrast to relatively minor changes before treatment. However, Dpd was more variable in its response, possibly partly because this marker was already low in several babies before the onset of treatment. Although we cannot exclude the possibility that a proportion of the changes we observed in the collagen markers following dexamethasone treatment might arise from altered clearance, this is unlikely to be a major factor as the collagen markers have different clearance mechanisms: PICP and P3NP are large molecules, both cleared by liver endothelial cells but via different receptors (21), whereas ICTP is a small molecule largely cleared by the kidney, where it presumably contributes to urinary pyridinium cross-link excretion. The positive relationship between ICTP and Pyd therefore makes it unlikely that the low plasma ICTP arose from increased renal clearance.

It is well recognized that glucocorticoids inhibit growth (6) by a direct action on the epiphyseal growth plate (22); they also inhibit chondrocyte proliferation $(23,24)$, osteoblast proliferation, and collagen synthesis $(7,8,25,26)$, but their effects on 


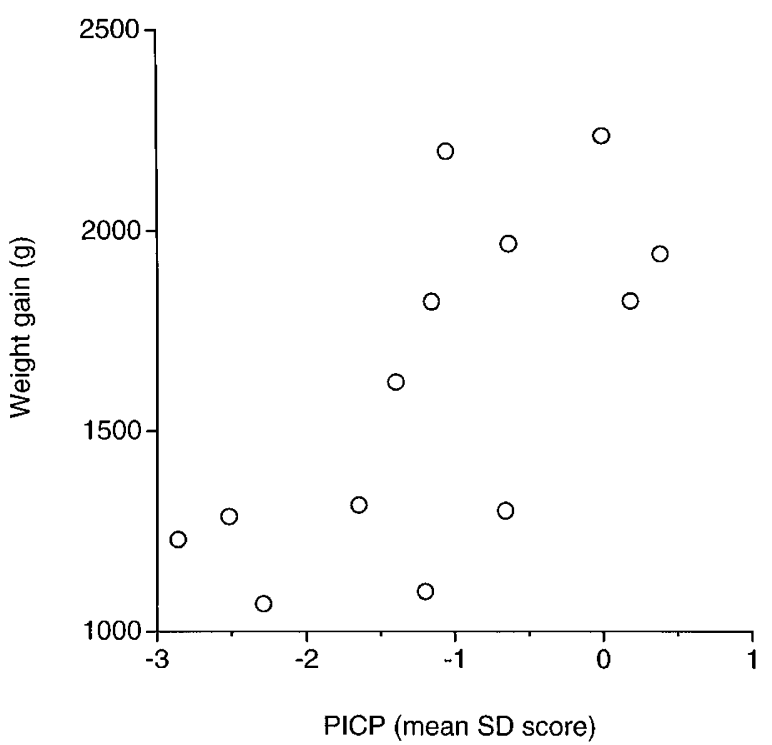

Figure 3. Total weight gain from day of birth to $\mathrm{d} 84$ versus PICP (expressed as mean SD score for each baby over the first $12 \mathrm{wk}$ of life); $r=0.69, p<$ 0.01 .

collagen degradation appear to be more controversial. This study confirms that both collagen synthesis and its degradation were markedly suppressed by dexamethasone, in keeping with the observed effects of glucocorticoids in children with asthma, inflammatory bowel disease, and acute lymphoblastic leukemia (12) and in agreement with a previous study on preterm infants treated with dexamethasone that reported reduced excretion of pyridinium cross-links (other markers were not measured) (10). In contrast to the effects of dexamethasone on collagen markers, we found that bone ALP was more variable in its response, but generally increased slightly. This was probably because glucocorticoids down-regulate osteoblast proliferation (resulting in a decrease in PICP), causing them to differentiate prematurely and express increased amounts of ALP $(8,25-27)$. Later, ALP may fall because of reduced recruitment of osteoblasts and chondrocytes from the proliferative phase and narrowing of the hypertrophic layer of the growth plate $(24,28)$. Although the babies in our study were supplemented with mineral, any mineral deficiency might further modulate the bone ALP response to dexamethasone.

No studies have yet been conducted to establish the optimum dosage of dexamethasone in preterm neonates. Such studies might be facilitated if there were reliable markers that would reflect rapidly the immediate effects of dexamethasone on growth. Simple measurements of weight may be misleading in this context as marked fluctuations occur owing to steroidinduced diuresis. On a group basis, we found strong negative correlations between all collagen markers and dexamethasone dose. Most individual babies also showed strong negative correlations between markers and dose throughout their treatment course; these did not appear to be due simply to a waning of response with time, because increasing the dose during a treatment course resulted in a renewed decrease in markers. However, the slopes of the regression lines differed by a factor of two among babies and a few babies showed a weaker relationship with dose. There therefore seemed to be differ- ences among babies in their susceptibility to the adverse effects of dexamethasone on bone turnover and growth.

Different individuals may vary in the degree to which they experience adverse side-effects from glucocorticoid treatment. We have shown that the cumulative dexamethasone dose was not helpful in predicting either weight gain or linear growth. However, PICP (expressed as the mean SD score for each infant, integrated over the first $12 \mathrm{wk}$ of life) was strongly and positively correlated with weight gain at $12 \mathrm{wk}$, accounting for half its variability. The evidence therefore suggests that the effects of dexamethasone on growth may be mediated to a considerable extent through suppression of type I collagen synthesis, as measured by PICP.

We conclude that dexamethasone causes a profound suppression of collagen turnover in preterm infants, that the suppression is dose-related but differs in magnitude among individual babies, and that the degree of suppression of type I collagen synthesis contributes significantly the outcome of weight gain over the first 3 mo of life.

Acknowledgments. The authors thank the Medical Research Council, Pharmacia and Upjohn, and Milupa for their support of this study.

\section{REFERENCES}

1. Ng PC 1993 The effectiveness and side-effects of dexamethasone in preterm infants with bronchopulmonary dysplasia. Arch Dis Child 68:330-336

2. Jones R, Wincott E, Elbourne D, Grant A 1995 Controlled trial of dexamethasone in neonatal chronic lung disease: a 3-year follow-up. Pediatrics 96:897-906

3. Yeh TF, Lin YJ, Huang CC, Chen YJ, Lin CH, Lin HC, Hsieh WS, Lien YJ 1998 Early dexamethasone therapy in preterm infants: a follow-up study. Pediatrics 101:E7

4. Wales JKH, Milner RDG 1988 Variation in lower leg growth with alternate day steroid treatment. Arch Dis Child 63:981-983

5. Wolthers OD, Pedersen S 1990 Short term linear growth in asthmatic children during treatment with prednisolone. Brit Med J 301:145-148

6. Tönshoff B, Jux C, Mehls O 1996 Glucocorticoids and growth. In: Kelnar CJH (ed) Baillière's Clinical Paediatrics-Paediatric Endocrinology. Baillière Tindall, London, pp 309-331

7. Gennari C 1994 Glucocorticoid induced osteoporosis. Clin Endocrinol 41:273-274

8. Canalis E 1996 Mechanisms of glucocorticoid action in bone: implications to glucocorticoid-induced osteoporosis. J Clin Endocrinol Metab 81:3441-3447

9. Gibson AT, Pearse RG, Wales JKH 1993 Growth retardation after dexamethasone administration: assessment by knemometry. Arch Dis Child 69:505-509

10. Weiler HA, Paes B, Shah JK, Atkinson SA 1997 Longitudinal assessment of growth and bone mineral accretion in prematurely born infants treated for chronic lung disease with dexamethasone. Early Hum Dev 47:271-286

11. Shrivastava A, Lyon AJ, McIntosh N 2000 The effect of dexamethasone on growth, mineral balance and bone mineralisation in preterm infants with chronic lung disease. Eur J Paediatr 159:380-384

12. Crofton PM, Kelnar CJH 1998 Bone and collagen markers in paediatric practice. Int J Clin Pract 52:557-565

13. Crofton PM, Shrivastava A, Wade JC, Stephen R, Kelnar CJH, Lyon AJ, McIntosh N 1999 Bone and collagen markers in preterm infants: relationship with growth and bone mineral content over the first 10 weeks of life. Pediatr Res 46:581-587

14. Melkko J, Niemi S, Risteli L, Risteli J 1990 Radioimmunoassay of the carboxyterminal propeptide of human type I procollagen. Clin Chem 36:1328-1332

15. Risteli J, Niemi S, Trivedi P, Mäentausta O, Mowat AP, Risteli L 1988 Rapid equilibrium radioimmunoassay for the amino-terminal propeptide of human type III procollagen. Clin Chem 34:715-718

16. Risteli J, Elomaa I, Niemi S, Novamo A, Risteli L 1993 Radioimmunoassay for the pyridinoline cross-linked carboxy-terminal telopeptide of type I collagen: a new serum marker of bone collagen degradation. Clin Chem 39:635-640

17. Crofton PM 1987 Properties of alkaline phosphatase isoenzymes in plasma of preterm and term neonates. Clin Chem 33:1778-1782

18. Crofton PM, Hume R 1987 Alkaline phosphatase isoenzymes in the plasma of preterm and term infants: serial measurements and clinical correlations. Clin Chem 33:1783-1787

19. Pratt DA, Daniloff Y, Duncan A, Robins SP 1992 Automated analysis of the pyridinium crosslinks of collagen in tissue and urine using solid-phase extraction and reversed-phase high-performance liquid chromatography. Anal Biochem 207:168175 
20. Black D, Duncan A, Robins SP 1988 Quantitative analysis of the pyridinium crosslinks of collagen in urine using ion-paired reversed-phase high performance liquid chromatography. Anal Biochem 169:197-203

21. Smedsrød B, Melkko J, Risteli L, Risteli J 1990 Circulating C-terminal propeptide of type I procollagen is cleared mainly via the mannose receptor in liver endothelial cells. Biochem J 217:345-350

22. Baron J, Huang Z, Oerter KE, Bacher JD, Cutler GB 1992 Dexamethasone acts locally to inhibit longitudinal bone growth in rabbits. Am J Physiol 263:E489E492

23. Hill DJ 1981 Effects of cortisol on cell proliferation and proteoglycan synthesis and degradation in cartilage zones of the calf costochondral growth plate in vitro with and without rat plasma somatomedin activity. J Endocrinol 88:425-435
24. Robson H, Anderson E, Eden OB, Isaksson O, Shalet S 1998 Chemotherapeutic agents used in the treatment of childhood malignancies have direct effects on growth plate chondrocyte proliferation. J Endocrinol 157:225-235

25. Oikarinen AI, Uitto J, Oikarinen J 1986 Glucocorticoid action on connective tissue: from molecular mechanisms to clinical practice. Med Biol 64:221-230

26. Delaney AM, Dong Y, Canalis E 1994 Mechanisms of glucocorticoid action in bone cells. J Cell Biochem 56:295-302

27. Stein GS, Lian JB, Owen TA 1990 Relationship of cell growth to the regulation of tissue-specific gene expression during osteoblast differentiation. FASEB J 4:3111-3123

28. Brown RA, Rees JA, Mcfarland CD, Lewinson D, Ali SY 1990 Microvascular invasion of rabbit growth plate cartilage and the influence of dexamethasone. Bone Miner 9:35-47 